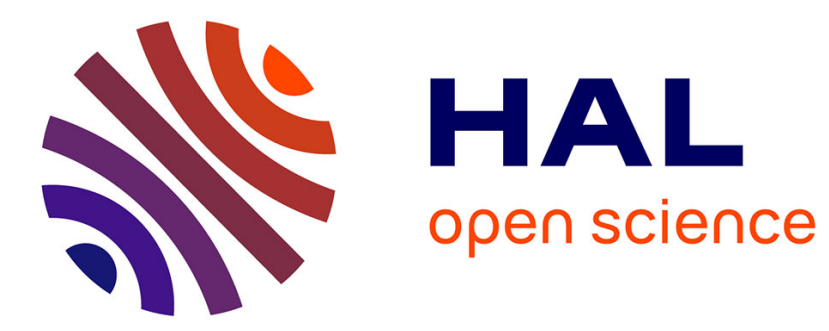

\title{
Le corps, un témoin? Psychanalyse et différence des sexes
}

Thamy Ayouch

\section{To cite this version:}

Thamy Ayouch. Le corps, un témoin? Psychanalyse et différence des sexes. Recherches en psychanalyse, 2013, 15 (1/2013), pp.49-59. halshs-01003788

\section{HAL Id: halshs-01003788 \\ https://shs.hal.science/halshs-01003788}

Submitted on 11 Jun 2014

HAL is a multi-disciplinary open access archive for the deposit and dissemination of scientific research documents, whether they are published or not. The documents may come from teaching and research institutions in France or abroad, or from public or private research centers.
L'archive ouverte pluridisciplinaire HAL, est destinée au dépôt et à la diffusion de documents scientifiques de niveau recherche, publiés ou non, émanant des établissements d'enseignement et de recherche français ou étrangers, des laboratoires publics ou privés. 


\title{
Le corps, un témoin ? Psychanalyse et différence des sexes
}

\author{
The Body of Evidence. Psychoanalysis and Sex Difference
}

\author{
Thamy Ayouch ${ }^{1}$ \\ Un témoignage des langues
}

C'est historiquement en référence à l'objet matériel, visible et attestable que le terme de témoin apparaît en ancien français. Renvoyant à " ce qui sert de preuve », au document, en 1165, il désigne seulement dans un second temps la "personne qui a vu, entendu quelque chose et peut le certifier ».

L'usage actuel distingue ainsi, selon le Trésor, les sens de personne ou de chose : le témoin est un spectateur, assistant silencieusement à un événement, ou la "personne qui certifie ou qui peut certifier ce qu'elle a vu ou entendu ». En justice, le témoin permet de faire procéder à l'application de la loi, ou d'attester l'exactitude et l'authenticité des identités et des déclarations.

Toutefois, c'est également le sens de chose qui affleure dans ce vocable. Preuve matérielle, indice, pièce à conviction, le témoin peut être muet, sous la forme d'un objet ou d'un lieu qui porte témoignage d'un événement, ou éloquent, lorsqu'il permet de constater et vérifier, à la manière d'une base de comparaison dans une expérience scientifique, ou du bâton que se transmettent les coureurs dans une course de relais.

Ces différents sens ont donc directement trait à une visibilité, une tangibilité ou une audition, autant d'attestations sensibles qui lient le témoin à la perception par le corps, et à la perception des corps - substances étendues - et du corps.

Cette inscription dans le corps n'est pas absente de l'origine latine du terme, testis-is, révélant une homonymie entre le témoin, et le petit témoin, testis ou testiculus, le testicule. Le témoin fait référence directe à un gage de masculinité : le testicule est témoin du sexe mâle. L'anatomie est toutefois ici prise dans le réseau symbolique du juridique : ne témoignent, d'une lignée, que les hommes, probablement, selon une autre hypothèse, en jurant sur ce qu'ils auraient de plus cher.

Mais de quoi le testicule est-il véritablement témoin ? Cette particularité anatomique du corps masculin donne à voir, au même titre que la saillance du pénis, la différence anatomique des sexes. Le, ou, de préférence, les testicules renvoient à l'un des deux sexes de l'espèce, inscrivent le mâle dans sa distinction de la femelle. Etrange

1 Psychanalyste, Maître de Conférence en psychopathologie clinique, Université Lille 3, Chercheur, Université Paris 7, Professor Visitante do Exterior, Universidade de São Paulo. 
témoignage du corps pour la masculinité, dans les langues latines, là où une langue sémitique, l'hébreu, renvoie le témoin à l'autre sexe.

En hébreu, témoin se dit עד [ed], et témoignage עדות [edot]. Si le vocable recouvre bien des sens du testis latin, c'est toutefois en s'en différenciant particulièrement eu égard au corps : le second sens de עד tel qu'il apparaît dans le dictionnaire Even Shoshan, fait référence au linge utilisé par la femme menstruée pour absorber le sang des règles ${ }^{2}$. Le témoin atteste ici de la non-grossesse, inscrivant, la féminité comme potentielle maternité, et l'en distinguant en même temps. Le corps est témoin d'une différence anatomique assignant un sexe, une potentialité en creux - la maternité - et la certitude d'une transmission, celle du judaïsme.

La constitution du témoin est alors affaire de connaissance, toutefois liée à de l'inconnaissable. En effet, עד [ed] articule un ע [ayin] à un ד [dalet]. La première lettre, ע désigne l'œil, le regard et la source : aux sens originaires de voir et de consulter, elle ajoute ceux d'apparaître et de disparaître, permettant ainsi la transition d'un intérieur à un extérieur, des profondeurs de la terre à la visibilité de la surface, comme le fait la source par le passage de l'eau. La seconde lettre, r, désigne la porte et l'ouverture, notamment triangulaire, et, partant, aussi le sexe ou le sein de la femme ${ }^{3}$.

Le témoin est ainsi cet œil qui se tient au seuil, à la surface du sexe, et atteste d'un passage de l'intérieur à l'extérieur. Il est cette ouverture du corps féminin donnant à voir un invisible. Mais l'ouverture est également celle de l'œil: le témoin est celui que caractérise le dessillement. C'est cette étymologie qu'il aurait par צרזף [tserouf], permutation des lettres, en partage avec la connaissance, ידיעה, [yediaa] et le connaître, dans son sens biblique, ידע [yedaa]. Connaître revient donc à outrepasser l'inaction, contemplative, du témoin pour traverser le seuil : à agir sur ce départage du visible et de l'invisible, de l'intérieur et de l'extérieur, dans un rapport charnel ${ }^{4}$.

Surgit donc la question de savoir si le corps témoigne aussi directement d'une assignation de sexe, masculin, ou féminin et comment cela s'effectue. Les réseaux du voir, du toucher, de l'entendre ou de l'attestation sensible ne correspondent pas, la psychanalyse le souligne bien, à l'immédiateté naturelle d'un percevoir, mais à la multiplicité d'inscriptions imaginaires et symboliques, où il n'est de corps que psychisé et symbolisé. Seul un certain type de symbolisation de son désir permet au sujet de percevoir son corps, qui ne se réduit pas à la somme d'innervations brutes, mais s'aborde toujours à partir d'une structuration du fantasme.

Poser la question du corps comme témoin revient à demander à quoi le corps assiste ici, s'il doit en rendre compte ou est témoin muet, s'il compte comme pièce à conviction dans l'inscription de la différence sexuelle ou témoigne d'une idéologie. Soulever cette question ne manque pas d'interroger certaines notions analytiques de la sexuation et de

\footnotetext{
2 Je remercie Keren Gitai de m'avoir confirmé cette étymologie.

${ }^{3}$ Ouaknin, M. A. (1997). Les mystères de l'alphabet. Paris: Assouline.

${ }^{4}$ Je remercie ici vivement Esther et Laurent Picard pour leurs analyses précieuses. Ceux-ci m'indiquaient également que la gematria du témoin, עי עי somme de la valeur numérique de ses lettres, est de 74 (le ayant une valeur de 70, le 7 de 4), même chiffre désignant la somme des lettres de la lettre $ל,(\zeta, \square$, et 7 équivalent à 74), lettre symbolisant l'étude et la connaissance. Le paradigme du témoin, outre le connaître, serait alors l'étude.
} 
la sexualité. C'est demander de quelle articulation à la visibilité des corps la différence des sexes procède, quelle inscription de l'ordre symbolique elle permet, et comment elle peut être, par la théorie, assignée. Il s'agit d'interroger le trajet du corporel au sexué, puis du sexué au sexuel, en examinant à nouveau frais si l'anatomie est véritablement un destin.

\section{L'anatomie, un destin?}

La notion freudienne de sexualité infantile, perverse polymorphe, ne manque pas d'introduire, dès la première édition des Trois essais sur la théorie sexuelle, une inédite dé-biologisation de la sexualité et de la sexuation. S'ouvrant en effet par les "Aberrations sexuelles », qui inscrivent la sexualité contre l'opinion populaire la liant à la reproduction, cet ouvrage détache la pulsion de tout attrait de l'objet ${ }^{5}$, position que confirme le texte "Pulsions et destin des pulsions ${ }^{6}$. En ce sens, le sexuel-infantile, conçu comme "gain de plaisir » irréductible à la satisfaction d'une fonction vitale, dans sa dimension perverse polymorphe, se retrouve comme composante du désir et de la pratique sexuelle, qui n'en est toutefois qu'une des manifestations parmi d'autres.

Cette dé-naturalisation de la sexualité, détachée de la reproduction, ne manque pas de dé-biologiser la sexuation. En effet, lorsque la visée de la pratique sexuelle n'est pas le destin biologique de la reproduction, la complémentarité des deux sexes de l'espèce apparaît toute relative.

Plus encore que la relativité d'une complémentarité des sexes, c'est essentiellement leur définition qui semble problématique. Freud souligne en effet à plusieurs reprises que si les notions de «masculin» et de «féminin» sont "peu équivoques pour l'opinion commune », elles restent de part en part «confuses » ${ }^{7}$. La psychanalyse «ne peut (...) élucider » l'essence de ces notions, « elle reprend à son compte les concepts et les mets à la base des ses travaux $»^{8}$. Cette différence des sexes est déclarée ténue ; elle se soutient d'une autre opposition qui n'est pas suffisante :

«Si l'on tente de les ramener à des principes plus originaires, la masculinité se volatilise en activité, et la féminité en passivité, ce qui est trop peu »9.

Si Freud évoque l'articulation inversée des complexes d'CEdipe et de castration, chez la fille et chez le garçon, il n'en souligne pas moins que les vicissitudes oedipiennes des individus des deux sexes «sont composées à la fois de traits masculins et de traits

\footnotetext{
5 « Il est probable, écrit Freud, que la pulsion sexuelle est d'abord indépendante de son objet et que ce ne sont pas davantage les attraits de ce dernier qui déterminent son apparition ", Freud, S. (1987). Trois essais sur le théorie sexuelle. Paris: Gallimard, Folio Essais, p 54.

6 « Il est ce qu'il y a de plus variable dans la pulsion, il ne lui est pas originairement lié : mais ce n'est qu'en raison de son aptitude particulière à rendre possible la satisfaction qu'il est adjoint », Freud, S. (1968). Pulsions et destins des pulsions. Dans S. Freud, Métapsychologie. Paris: Gallimard, Folio Essais.

7 Freud, S. (1987). Trois essais sur le théorie sexuelle. Paris: Gallimard, Folio Essais.

${ }^{8}$ Freud, S. (1973). Psychogénèse d'un cas d'homosexualité féminine. Dans S. Freud, Névrose, psychose, perversion. Paris: P.U.F., p 270.

${ }^{9}$ Ibid.
} 
féminins ${ }^{10}$, et tous les individus humains, "par suite de leur constitution bisexuelle et de leur hérédité croisée, possèdent à la fois des traits masculins et des traits féminins, si bien que le contenu des constructions théoriques de la masculinité pure et de la féminité pure reste incertain ${ }^{11}$.

Est-ce à dire que le corps, dans cette distinction anatomique que soutient faiblement l'opposition activité/passivité, ne témoigne que peu de la différence des sexes ?

La théorie freudienne est loin d'être univoque à ce sujet, et le témoignage du corps semble prendre ici une importance toute particulière dans la définition psychique de la sexuation et de la sexualité. Freud écarte la "réclamation féministe d'une égalité des droits entre les sexes », qui oublie trop vite que "la différence morphologique [doit] se manifester dans des différences dans le développement psychique ${ }^{12}$. Et de conclure, parodiant Napoléon : « l'anatomie est un destin »13.

La tendance semble radicalisée en 1937, lorsque, dans "Analyse avec fin, analyse sans fin », il souligne que la source la plus puissante de la résistance, en analyse, reste, pour les hommes, la révolte contre l'attitude passive ou féminine à l'endroit d'un autre homme, et pour les femmes l'envie du pénis, aspiration à la possession d'un organe génital masculin ${ }^{14}$. La biologie et son déterminisme, auparavant écartés, semblent ici de nouveau convoqués :

"On a souvent l'impression, avec le désir de pénis et la protestation virile, de s'être frayé un passage, à travers toute la stratification psychologique, jusqu'au "roc d'origine » et d'en avoir ainsi fini avec son travail ; Il ne peut pas en être autrement, car pour le psychique le biologique joue véritablement le rôle du roc d'origine sous-jacent. Le refus de la féminité ne peut évidemment rien être d'autre qu'un fait biologique, une part de cette grande énigme de la sexualité ${ }^{15}$.

Reste à déterminer la valeur de cette implication du biologique, garante d'une différence des sexes, liée ici au témoignage direct d'une différence d'organes : le biologique, dans son expression anatomique, est-il le dernier mot de la différence des sexes, ou n'intervient-il ici que comme l'index d'une énigme, celle, irréductible, de la sexualité ?

En outre, que penser de la définition classique introduite dans L'Homme Moïse et le monothéisme, et renvoyant la sexuation à un platonisme peu innovant où le masculin est esprit, intelligible, et le féminin devenir sensible ? Assignant ainsi l'homme et la femme à des rôles sexuels spécifiques, la psychanalyse freudienne n'introduit-elle pas une normativité, qui, par delà la spécificité du champ de l'inconscient, convoque des prescriptions sociales déterminantes?

\footnotetext{
10 Ibid., p 129.

11 Ibid., p 132.

12 Freud, S. (1969). La disparition du complexe d'Oedipe. Dans S. Freud, La Vie sexuelle. Paris: P.U.F., p 121.

13 Ibid.

14 Freud, S. (1985). L'analyse avec fin et l'analyse sans fin. Dans S. Freud, Résultats, idées, problèmes. II. Paris: P.U.F., p 267.

15 Ibid., p 268.
} 
Que comprendre alors du rôle du corps comme témoin? Ne serait-il qu'un précaire témoin de la différence des sexes, qui apparaît elle-même liée à la mince opposition de passivité/activité, ou à celle, convenue, de l'intelligible et du sensible, plus qu'à celle du masculin/féminin dont témoigneraient les organes?

La perspective de Monique Schneider, dans Généalogie du masculin, Le paradigme du féminin, et une série d'articles divers, souligne, avec minutie et érudition, les nuances du texte freudien à ce sujet.

\section{Par delà le binaire : diversité des sexes et dans les sexes}

Selon Monique Schneider, la différence sexuelle ne saurait faire l'objet d'un seul partage binaire dans la théorisation freudienne. En évoquant les cellules germinales contre la pulsion de mort, dans "Au delà du principe de plaisir », Freud semble dépasser la logique d'un avoir/ou n'avoir pas de pénis déterminant la sexuation de l'enfant puis de l'adulte pour introduire la «turbulence théorique », propre à l'Eros, de partenaires de la rencontre sexuelle à la fois différents et ressemblants ${ }^{16}$.

Nous déduirions de cela que cette différence définie par la binarité, et dont le corps se fait témoin, est celle, toute visible, d'une captation imaginaire: elle provient d'une théorie sexuelle infantile du garçon qui, comparant, par le regard la "région génitale » de la petite fille, introduit l'alternative d'un avoir ou n'avoir pas.

Autre est la perspective, selon Monique Schneider, lorsque la sexuation masculine est envisagée par delà cette mise en scène comparative. Traitant de l'érection dans «Pour introduire le narcissime », Freud écrit :

« Nous connaissons le modèle d'un organe douloureusement sensible, modifié en quelque façon sans être pourtant malade au sens habituel ; c'est l'organe génital en état d'excitation. Il est alors congestionné, turgescent, humide, et le siège de sensations diverses ${ }^{17}$.

Cet organe congestionné, Blutdurchströmt, traversé par un fleuve de sang, souligne Monique Schneider, apparaît ici dans une étrange proximité avec l'organe féminin ${ }^{18}$ : la différence des sexes, lorsqu'elle excède la binarité de la comparaison par le regard, est à la fois dissemblance et ressemblance.

Apparaît alors une véritable féminisation du masculin dans bien des passages de l'œuvre freudienne. Certes, la visée comparative définit la femme par ce qui, distinct du «morceau » chéri dont jouit le corps masculin, fait l'objet d'un manque. Mais le corps de la différence anatomique s'avère ici, à travers un regard qui reste au seuil (comme le note l'étymologie de עיד), doublement témoin dans le sens hébraïque.

\footnotetext{
${ }^{16}$ Monique Schneider « La pulsion de mort et la différence sexuelle », Analyse Freudienne Presse2/2002 (no 6), p. 2940.

17 Freud, S. (1969). Pour introduire le narcissisme. Dans S. Freud, La Vie sexuelle. Paris: P.U.F., p 90.

18 Monique Schneider « La pulsion de mort et la différence sexuelle », Analyse Freudienne Presse2/2002 (no 6), p. 2940.
} 
1. Il est d'abord annulation du testis latin. Dans la Généalogie du masculin, Monique Schneider souligne la mise à l'écart des testicules de la définition freudienne de la masculinité, similaire à l'omission par le regard de l'enfant de cette partie de l'appareil génital :

" Il est d'ailleurs frappant, écrit Freud dans "L'organisation génitale infantile », de constater combien peu d'attention suscite chez l'enfant l'autre partie de l'organe génital masculin, les bourses, avec ce qui leur est attaché. D'après les analyses, on ne pourrait pas deviner que quelque chose d'autre que le pénis appartient aussi à l'organe génital » (Freud S., 1923, p. 187).

Les bourses, petit sac, procèderaient d'une féminité ${ }^{19}$, liée à la fécondité, à la fois dans les théories sexuelles infantiles, et dans une répartition sociétale séparant l'accès au pouvoir du champ de la fécondité et la paternité. Ainsi rappelle-t-elle que le pluriel patres s'oppose aux proletarii, définis par Alain Rey comme « ceux qui mettent au monde des enfants $»^{20}$, dans une classification sociétale proche de la hiérarchie tripartite attribuée par Dumézil à la civilisation indo-européenne : prêtres, guerriers, producteurs.

Evoquant Françoise Héritier dans Masculin/Féminin. La pensée de la différence (Paris, Odile Jacob, 1996), Monique Schneider conclut que ce n'est pas le sexe mais la fécondité qui ferait ainsi la différence entre masculin et féminin. Précisons toutefois que la perspective de Monique Schneider reste très distincte de celle de Françoise Héritier, qui fait de la butée indépassable de la différence des sexes le modèle de toute différence, et le point de structuration de toute pensée, là où Monique Schneider semble lui substituer une diversité.

Observons donc que la différence apparaît ici au sein du même sexe, et met en perspective le pouvoir d'un côté, et la fécondité de l'autre. Si Freud établit une « logique du glaive » et rejoint parfois une conception socialisée de la différence des sexes, il n'en interroge pas moins la pensée binaire en introduisant une proximité entre les sexes. Ainsi le «rêve du grand exploit » commenté par Freud dans L'Interprétation des rêves, et rapporté par M. Schneider, fait-il communiquer érection et accouchement du sexe :

«Le viril rêveur se voit allongé dans son lit; il est une femme enceinte. Cet état devient pour lui très pesant [...]. Derrière son lit est suspendue une carte géographique, dont le bord inférieur est maintenu tendu par une baguette de bois. Il arrache cette baguette en la prenant par les deux bouts, si bien qu'elle ne se brise pas en travers, mais se fend en deux moitiés dans le sens de la longueur. De cette manière, il s'est soulagé et il a favorisé l'accouchement ${ }^{21}$.

La grossesse et l'accouchement présentent ici un «chevauchement entre les marqueurs du masculin et du féminin ». C'est à nouveau à la fécondité du masculin que ce rêve est rapporté, lorsque Monique Schneider rappelle le lien qu'y voit Françoise Héritier avec la coutume de la subincision chez certaines peuplades, fente opérée par les hommes sur leur pénis pour devenir, dans cette féminisation, plus féconds.

\footnotetext{
19 Observons que dans divers dialectes de l'arabe médio-oriental, le sexe féminin est désigné par le sac, كيس.

20 Monique Schneider « Le corps masculin : une production culturelle ? », Champ psychosomatique 3/2010 (n 59), p. 15-29.

${ }^{21}$ Freud, S. (1957). L'interprétation des rêves. Paris: P.U.F., pp 352-353, rapporté par Monique Schneider dans «Surimpressions sexuelles », Cliniques méditerranéennes 2/2006 (n 74), p. 27-42.
} 
Les fonctions du masculin, souligne Monique Schneider, ne sauraient donc acquérir de valeur universelle : les diverses périodes historiques indiquent une alternance entre le masculin entendu comme un "ordre» ou comme une fécondité. Les répartitions de masculin et de féminin, à leur tour, ne concernent pas une différence des sexes, mais un chevauchement, un entrecroisement au sein du même sexe, "un vacillement dans les opérations visant à statuer sur la différence $»^{22}$.

2. Le deuxième sens d'un corps témoin dans l'acception hébraïque de עד, regard cantonné à la porte qu'il ne traverse pas, est lié à la signification de seuil qu'acquiert chez Freud le paradigme du féminin. C'est ici la thèse de l'ouvrage éponyme ${ }^{23}$, où Monique Schneider compare l'imprécision freudienne dans la nomination du sexe féminin, appelé le plus souvent "région génitale», à la prescription culturelle d'un devoir d'ignorance concernant le féminin originaire. Freud n'en développe toutefois pas moins l'image d'une fente, dès la fin des Etudes sur l'hystérie, caractérisant autant l'anatomie qu'un lieu psychique permettant l'admission (Aufnahme) du refoulé dans un rythme de fermeture-ouverture, qui remplace le schème masculin avancée-retrait :

« La topographie féminine, offrant cette 'fente étroite' qui donne accès à une demeure interne, va donc servir de paradigme pour figurer l'espace psychique, pouvant expulser ou accueillir diverses manifestations imprévisibles »24.

Le corps féminin offre ainsi le paradigme d'un espace disponible vide, mais curieusement délocalisé : cet habitacle interne féminin est refusé à la femme, pour être attribué au psychisme en général. Ne reste alors pour désigner le féminin que la forme externe de l'absence visible, et la sexualité féminine n'est globalement pensée par Freud qu'eu égard aux régions liminaires: clitoris, vagin. C'est ici une opération de désymbolisation du féminin liant le matriciel, l'interne, le retiré du regard, au cloaqual ${ }^{25}$.

Si le corps est ici témoin, c'est à travers ce regard posté au seuil : l'œil reste à la porte, comme l'indique le sens de ]ed[ עד Gageons que se produit ici un double mouvement, lorsque la théorie reprend l'interdit de connaître propre à la fascination horrifiée éprouvée devant le sexe féminin. Reproduisant, dans son mouvement, une théorie sexuelle infantile, lorsqu'elle appréhende la différence des sexes par le regard, elle produit une double captation imaginaire : c'est par le regard sur la « région génitale » de la fille, comparativement, que le garçon déduit la différence sexuelle, et que la théorie la cantonne à la binarité d'un avoir/ne pas avoir. Mais c'est aussi par ce regard qu'il assigne la fille à une sexuation que la théorisation ne manque pas de reproduire.

\section{Le témoignage muet du corps : la prescription du genre}

\footnotetext{
22 Monique Schneider «Surimpressions sexuelles », Cliniques méditerranéennes 2/2006 (no 74), p. 27-42.

23 Schneider, M. (2004). Le paradigme féminin. Paris: Aubier.

24 Ibid., p 99.

25 Monique Schneider « Du cloacal au matriciel », Revue française de psychosomatique 2/2004 (n 26), p. 35-53.
} 
Comment s'effectue cette assignation d'une sexuation, à la fois par le regard de l'enfant et celui de la théorie? La perspective de Judith Butler, dans Troubles dans le genre, en rend compte, remodèle en profondeur les rapports du sexe et du genre et redistribue les sens de la différence des sexes. L'opposition entre sexe et genre n'est plus pensée sur le modèle du divorce entre nature et culture, ce qui, en ultime recours, procède toujours à la fondation d'une construction sociale sur un substrat d'identité biologique, naturalisant alors la différence des sexes pour historiciser celle des genres. Pas moins que le genre, le sexe est lui aussi une construction sociale : l'anatomie n'est pas un destin mais une fabrication historique ${ }^{26}$.

Pour Judith Butler, il n'est pas de nature ontologique, de différence des sexes anatomique qui ne soit toujours et déjà prise dans le sens institué, construit, culturellement et historiquement défini, du genre. Le genre désigne alors précisément l'appareil de production et d'institution des sexes eux-mêmes: il est l'ensemble des moyens discursifs et culturels par lesquelles une "'nature sexuée' ou un 'sexe naturel' est produit et établi dans, un domaine 'prédiscursif' qui précède la culture, telle une surface politiquement neutre sur laquelle intervient la culture après coup ${ }^{27}$.

Le corps ne serait alors témoin de rien du tout, il n'indiquerait aucun substrat naturel de la différence sexuelle, puisqu'il n'apparaît que comme construit culturel mis en forme, érigé et modelé par des assignations de genre qui produisent le sexe. Il ne s'agit toutefois pas, comme l'indique J. Butler dans Ces corps qui comptent ${ }^{28}$, de nier la matérialité du corps, mais de l'aborder non comme réalité préalable mais comme effet réel des régulations sociales et des assignations normatives.

Le genre, et le sexe qu'il produit, s'avèrent alors performatifs, dans le sens austinien : les actes, gestes, désirs exprimés et réalisés créent l'illusion d'un noyau interne, maintenue précisément par une répétition constante de la norme. C'est une répétition singulière, qui crée, par l'imitation renouvelée, l'idée d'un modèle original. Celui-ci n'existerait pourtant pas, mais découlerait précisément de cette performativité. Le genre étant créé par les performances sociales ininterrompues, l'idée d'un sexe essentiel s'inscrit dans la même stratégie de dissimulation du caractère performatif du genre ${ }^{29}$. La performativité du genre et la production du sexe dont elle décide n'est toutefois pas un choix délibéré, mais une interpellation sociale : elle n'est pas un acte subjectif isolé mais une réitération collective, une assignation normative.

Le genre n'est plus alors opposé au sexe, mais il le produit, en instaurant des normes qui traversent le sexe et la sexualité dans une véritable inscription corporelle définie comme «mélancolie de genre».

\footnotetext{
26 Thomas Laqueur le montre déjà dans Laqueur, T. (1992). La Fabrique du sexe. Essai sur le corps et le genre en Occident. Paris: Gallimard.

${ }^{27}$ Butler, J. (2005). Troubles dans le genre. Le féminisme et la subversion de l'identité. Paris: La découverte, p 69.

${ }^{28}$ Butler, J. (2009). Ces corps qui comptent. De la matérialité et des limites discursives du "sexe". Paris: Amsterdam.

${ }^{29}$ Ibid., p 266.
} 
Concluons ici que le corps est un témoin muet: il ne vaut pas comme marque, indice, pièce à conviction, dans lesquels s'attesterait une inscription sexuée. Il assiste, en spectateur silencieux et oublieux, à cette mélancolie de genre qui dessine sa surface, sa sexuation et son désir.

\section{Psychanalyse et genre}

Ne conviendrait-il pas alors de penser davantage le rapprochement entre théories du genre et psychanalyse, dans cette tentative de cerner l'opérativité des différences de genre dans la sexuation ? C'est, semble-t-il, une intersubjective, et partant, toute sociale différence des genres qui ici opère dans l'assignation d'une sexuation. Comme le souligne Jean Laplanche dans Castration, symbolisations, préalable à la différence des sexes se trouve une différence des genres, du masculin et du féminin, admise par la psychanalyse sans être théorisée. Là où l'enfant, plongé dans un univers adulte reçoit sans la questionner l'opposition sociale transmise du masculin/féminin, la psychanalyse en rend compte de manière essentialisée, par sa "tendance à situer la distinction masculin- féminin comme la distinction tout à fait terminale, celle à laquelle on doit arriver $»^{30}$.

Cette différence reste toutefois, comme le développe Jean Laplanche dans un texte ultérieur, assignée par le social :

« Le genre, à mon sens pour le définir, le terme capital, je ne suis pas le seul à le dire d'ailleurs, c'est celui d' 'assignation'. Assignation souligne le primat de l'autre dans le processus. [...] L'assignation est un ensemble complexe d'actes qui se prolonge dans le langage et dans les comportements significatifs de l'entourage. On pourrait parler d'une assignation continue ou d'une véritable prescription. Cette idée d'assignation ou d' 'identification comme', change complètement le vecteur de l'identification. Je pense ici qu'il y a ici une façon de sortir de l'aporie de cette si 'belle' formule de Freud qui a fait tellement cogiter et commenter : 'l'identification primitive au père de la préhistoire personnelle'. [...] Ne serais-ce pas, plutôt qu'une 'identification à', une 'identification par'. En d'autres termes je dirais : 'identification primitive par le socius de la préhistoire personnelle'»31.

Si le corps est témoin, dans cette captation du regard, il est alors, par l'inflation fantasmatique propre au voir, situant sous toute perception un irréductible imaginaire, ce qui cantonne une différence des genres, assignée par le socius primitif, à une différence des sexes toute binaire, définie par la logique de l'avoir/ne pas l'avoir.

Cette prévalence du genre, qui détermine une sexuation plus qu'elle n'en résulte, est ce qui semble être pointé par Jeanne lors de plusieurs séances. Cette jeune femme de 25 ans affirme, dès notre première rencontre, détester le vocable de "femme », qu'elle trouve indécent s'il lui est appliqué. Elle indique, par la suite, accepter en ultime recours celui de "fille », tout en refusant un ensemble d'activités qui lui sont associées. Cette désidentification du féminin conventionnel n'est toutefois affichée ni dans son style ni

\footnotetext{
30 Laplanche, J. (1980). Problématiques II. Castration, symbolisation. Paris: P.U.F., p 170.

${ }^{31}$ Laplanche, J. (2003). Sexual. La sexualité élargie au sens freudien. Paris: P.U.F, p 167.
} 
dans sa mise. Ayant entrepris des études de médecine qu'elle réussit avec brio, elle justifie son choix de la spécialité de traumatologie par une volonté de ne pas correspondre à ce qui d'elle serait attendu. Si une femme est plus susceptible de prendre la voie de la pédiatrie, pour son "instinct maternel», ce sera, dit elle, un «truc de garçon » dont elle s'appropriera dans la traumatologie. Les traumatologues sont de «fortes brutes» qui n'hésitent pas à «trancher dans le vif ». "Ils n'ont pas de sentiment», ajoute-t-elle, "c'est toujours plus simple de couper dans de la viande endormie que de parler à quelqu'un ».

Ses associations lors de cette séance l'amènent à développer une série d'oppositions : fille/garçon s'étaie, dans cette pratique du traumatologue, des distinctions gentille/cruel, faible/fort, et grosse/mince, cette dernière étant prégnante dans la détermination de ses comportements alimentaires. Elle resitue alors l'accusation de faiblesse de la troisième opposition dans les propos de son frère, prompt à l'en incriminer, ou à en qualifier tous ceux qui ne se montrent pas assez cruels.

Elle en vient ainsi à évoquer la naissance de ce premier frère, lorsqu'elle avait trois ans. Elle détestait ce nourrisson, «bout de viande braillard », qu'elle aurait tué si elle l'avait pu. Sitôt qu'il tentait de s'endormir, elle jetait des objets dans son berceau pour le réveiller. Mon intervention : " en somme, vous coupiez dans de la viande endormie » la laisse perplexe, probablement devant la similitude entre son désir d'être traumatologue, et le complexe d'intrusion dont il serait la sublimation.

Ici, la différence des sexes n'est qu'une distinction fonctionnant dans une série d'autres différences (grosse/mince, faible/fort, gentille/cruel), tout aussi instituées, et dont la base n'est pas tant la différenciation anatomique, que l'arrivée du premier puis du deuxième frère, détrônant Jeanne de la centralité de l'attention parentale. Ce n'est pas le Penis-neid ou le fantasme d'en avoir été privée qui détermine sa sexuation, mais l'assignation par des autres à une identification de genre contestée, car liée à une opposition à ses frères.

Fonder ainsi la différence des sexes sur une essentialisation de la nature, un « roc biologique », procède, hormis la captation imaginaire du regard du petit garçon et du théoricien posés à la seule lisière du sexe de la femme, d'une répétition des normes de genre, et crée un illusoire original du masculin et du féminin.

C'est pour lever toute dimension biologisante de la différence des sexes que Lacan développe sa théorie du phallus, distinct du pénis, signifiant d'un désir qui ne se structure pas de la différence anatomique des sexes. Nous n'aurons toutefois pas ici le loisir d'aborder la question du phallus comme semblant au fondement du rapport entre les deux sexes, ni celle formules de la sexuation du Livre XX du Séminaire, Encore. Si celles-ci établissent deux formes de jouissance (phallique et pas-toute phallique) départageant le masculin du féminin de manière non naturalisée, elles n'en posent pas moins la question de la légitimité, dans le raisonnement, d'un recours préalable à deux groupes sexués universels, "les hommes», "les femmes», et au mythe, sexué, de la horde primitive. 


\section{Conclusion}

La théorie analytique n'opère pas à la manière des autres théories. Elle convoque, dans sa lecture et dans son écriture, le travail en soi de l'analyse, n'est jamais conclue ou achevée bien qu'elle connaisse des moments de synthèse interprétative, et s'entend dans une relation à la praxis analytique, qui, plus qu'une simple technique, correspond à la rencontre de la singularité clinique et des transformations sociétales.

Ne conviendrait-il pas alors de prendre acte de la multiplicité des niveaux de la théorie? Si féminin et masculin sont relativisés, polysémiques, et dénaturalisés chez Freud, il n'en portent pas moins, par ailleurs, l'assignation d'identités historicisées d'homme et de femme, présentées comme roc biologique.

De quoi le corps est-il alors témoin? La différence ne peut être l'effet d'un simple constat, effectué par le corps percevant et à même le corps. Ce que l'expérience semble rencontrer n'est pas une différence, inscrite dans une binarité oppositive, mais une diversité de figures qui divergent ou convergent pour construire des sexuations. Les signifiants de la sexuation n'expriment pas une réalité ontologique, mais traduisent l'angle de perception de ressemblances et de dissemblances entre les sexes. Si l'on conçoit le sexe comme produit par le genre, la différence des sexes n'advient alors que d'une mise en relation particulière des corps, d'une opération interprétative.

De quoi le corps est-il alors témoin ? De ceci que la différence des sexes n'est pas la première, la principale ou l'unique qui structure la psyché : elle vient se signifier dans une série d'autres différences par lesquelles elle est produite. Le témoin muet de la construction et non d'une ontologique pré-donation de la différence des sexes serait ainsi le corps social dans lequel cette différence est produite.

La tâche de la psychanalyse n'est-elle pas alors de permettre une plasticité dans la construction de ces dissemblances et ressemblances, une créativité psychique en constant mouvement, par delà toute théorie binaire menacée par l'engourdissement ?

\section{Bibliographie}

Butler, J. (2009). Ces corps qui comptent. De la matérialité et des limites discursives du "sexe". Paris: Amsterdam.

Butler, J. (2005). Troubles dans le genre. Le féminisme et la subversion de l'identité. Paris: La découverte.

Freud, S. (1969). La disparition du complexe d'Oedipe. Dans S. Freud, La Vie sexuelle. Paris: P.U.F. 
Freud, S. (1985). L'analyse avec fin et l'analyse sans fin. Dans S. Freud, Résultats, idées, problèmes. II. Paris: P.U.F.

Freud, S. (1957). L'interprétation des rêves. Paris: P.U.F.

Freud, S. (1969). Pour introduire le narcissisme. Dans S. Freud, La Vie sexuelle. Paris: P.U.F.

Freud, S. (1973). Psychogénèse d'un cas d'homosexualité féminine. Dans S. Freud, Névrose, psychose, perversion. Paris: P.U.F.

Freud, S. (1968). Pulsions et destins des pulsions. Dans S. Freud, Métapsychologie. Paris: Gallimard, Folio Essais.

Freud, S. (1969). Quelques conséquences psychiques de la différence anatomique entre les sexes. Dans S. Freud, La Vie sexuelle. Paris: P.U.F.

Freud, S. (1987). Trois essais sur le théorie sexuelle. Paris: Gallimard, Folio Essais.

Lacan, J. (1966). Ecrits. Paris: Seuil.

Lacan, J. (1998). Le Séminaire, Livre IV. La Relation d'objet. Paris: Seuil.

Laplanche, J. (1980). Problématiques II. Castration, symbolisation. Paris: P.U.F.

Laplanche, J. (2003). Sexual. La sexualité élargie au sens freudien. Paris: P.U.F.

Laqueur, T. (1992). La Fabrique du sexe. Essai sur le corps et le genre en Occident. Paris: Gallimard.

Ouaknin, M. A. (1997). Les mystères de l'alphabet. Paris: Assouline.

Prokhoris, S. (2000). Le Sexe prescrit. La différence sexuelle en question. Paris: Aubier.

Roustang, F. (1985). Sur l'épistémologie de la psychanalyse. Dans M. Mannoni, Le Moi et l'autre. Paris: Denoël.

Schneider, M. (2004). Le paradigme féminin. Paris: Aubier. 